\title{
Ein Modell, welches die bei bestimmten Stellungen des Auges auftretende scheinbare Verzerrung eines Nachbildes anschaulich macht.
}

Von

Dr. Adolf Basler,

Privatdozent und Assistent am physiologischen Institut zu Tübingen.

(Mit 2 Textfiguren.)

Die Lehre von den Augenbewegungen gehört zu denjenigen Kapiteln der Physiologie, bei denen es schwierig ist, den Studierenden in knapper Form einen Überblick über die wesentlichen Daten zu geben. Die Schwierigkeiten vermehren sich noch, wenn es sich darum handelt, bei der von $\mathrm{Helmholtz}$ sorgfältig ausgearbeiteten Nachbildmethode sich vorzustellen, welche scheinbaren Veränderungen eines Nachbildes bedingt sind durch die Stellung der Angen, und welche durch die verschiedene Projektion auf eine ebene Wand. Deshalb habe ich zum leithteren Verständnis das im folgenden beschriebene Modell konstruiert, das den Zweck hat, den Einfluss der schiefen Projektion auf die Gestalt des Nachbildes zu zeigen, und an dem sich ausserdem die verschiedenen Begriffe, welche man zum Verständnis der Augenbewegungen geschaffen hat, studieren lassen.

Das Modell ist montiert auf einem festen Fuss $A$, in dessen Mitte auf einem vertikalen Zapfen sitzend und bei $B$ drehbar die Gabel $C C$ angebracht ist. Durch den oberen Teil ihrer Arme sind in horizontaler Richtung zwei Schrauben $D D^{1}$ ) eingelassen, welche als Achse dienen für einen Ring $E$. In diesem Ring ist um eine senkrechte Achse $F F$ drehbar eine runde Messingscheibe $G$ eingesetzt, welche einen durch den Drehpunkt des Auges gelegten Schnitt darstellen soll.

1) Auf Fig. 1 ist nur die eine Schraube mit $D$ bezeichnet. 
Als mittlerer Querschnitt der Netzhaut orier Netzhauthorizout wird die im Bulbus feste Ebene bezeichnet, welche bei der Primärstellung mit der Blickebene zusammenfällt. [Hering ${ }^{1}$ ) versteht darunter nur die Schnittlinie dieser Ebene mit der Netzhaut.]

Der mittlere Längsschnitt wird daroestellt durch die darauf senkrechte, ebenfalls im Bulbus festliegende, durch die Blicklinie gehende Ebene resp. ihre Schnittlinie mit der Netzhaut.

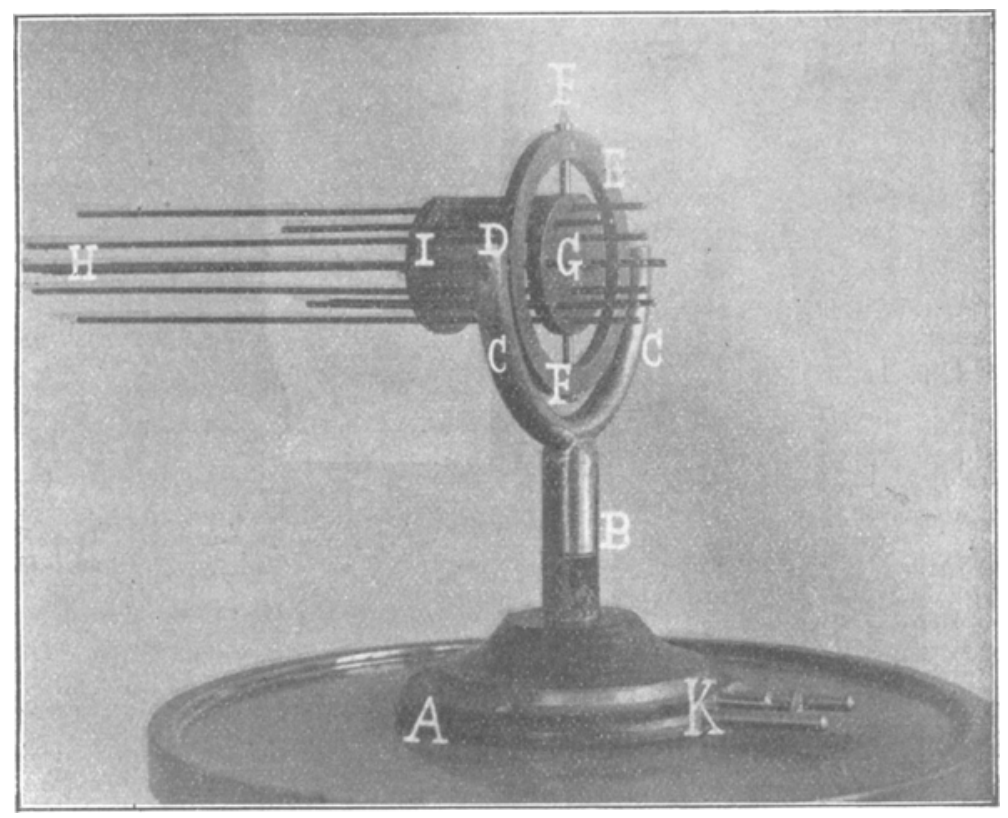

Fig. 1. Von der Seite gesehen.

Diese beiden Ebenen sind im Modell wiedergegeben durch zwei aufeinander senkrecht stehende Reihen von Stahlstäbchen $H$, welche in Kanälen im Bulbus stecken und darin verschoben werden können. Durch das mittlere Stäbchen wird gleichzeitig die Blicklinie markiert. Die hintere Messingscheibe $\mathcal{J}$ (Fig. 1), die mit der vorderen starr verbunden ist, hat nur den Zweck, die Führung für die Stähchen zu bilden. Durch ein an ibr angebrachtes System von Federn werden die Stäbe vor dem Ausfallen geschützt. Die Platte $J$ stellt gleich-

1) E. Hering, Der Raumsinn und die Bewegungen des Auges. Hermann's Handbuch der Physiologie Bd. 3 S. 343 (352). Leipzig 1879. 
Ein Modell, welches die bei bestimmten Stellungen des Auges etc. 325 zeitig die Retina dar, während man sich die Cornea vor der drehbaren Scheibe $G$ liegend zu denken hat.

Aus der Primärstellung kann das Auge bei in die Ferne gerichtetem Blick in jede andere Stellung übergehen, ohne dabei eine Rollung um die Blicklinie auszuführen. Man kann sich demnach jede sekundäre Steliung aus der primären so entstanden denken, dass das Auge sich um einen Durchmesser als Achse gedreht hat,

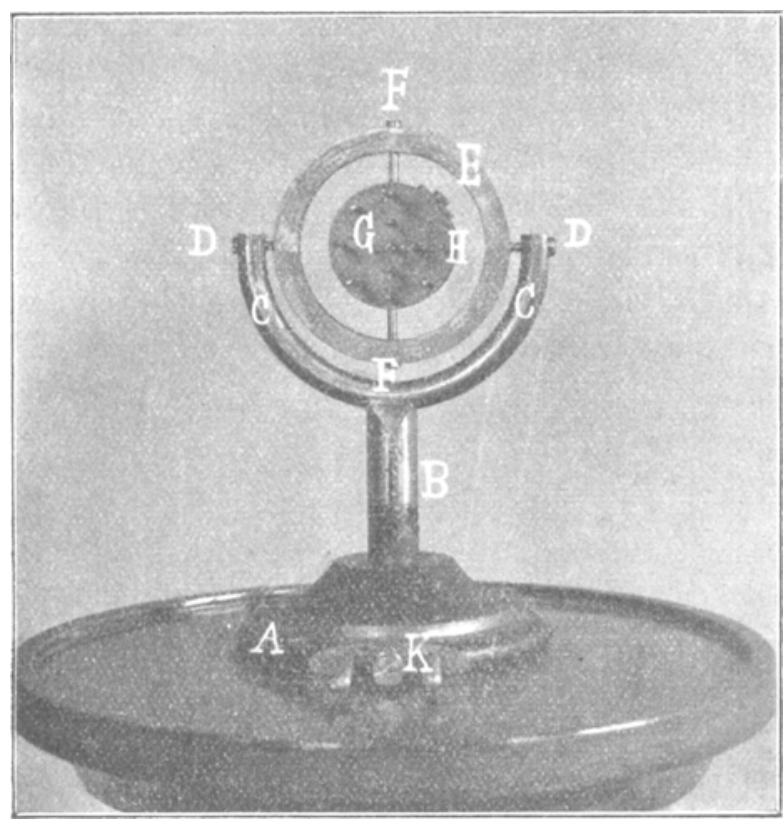

Fig. 2. Von vorn gesehen.

welcher zu der Blickrichtung sowohl in der primären als auch sekundären Stellung senkrecht steht.

Diese Tatsache, die von $\mathrm{Listing}^{1}$ ) theoretisch abgeleitet wurde und als Listing' sches Gesetz bezeichnet wird, wurde zuerst von Helm holtz ${ }^{2}$ ) experimentell bewiesen mit Hilfe der Nachbildmethode.

1) Veröffentlicht durch Th. Ruete, Lehrbuch der Ophthalmologie, 2. Aufl., Bd. 1 S. 37. Braunschweig 1853.

2) H. v. Helmholtz, Über die normalen Bewegungen des menschlichen Auges. Gräfe's Arch. f. Ophthalmologie Bd. 9 Abt. II S. 153 (172). 1863, und Handbuch der physiologischen Optik, 2. Aufl., S. 657. Hamburg und Leipzig 1896. 
Diese Methode beruht darauf, dass wir mit einem Auge in der Primärstellung einen Gegenstand, etwa ein Kreuz, fixieren, bis wir ein genügendes Nachbild hahen, und dann mit dem Auge eine bestimmte Bewegung ausführen. Dabei macht sich aber der Umstand bemerkbar, dass bei dem Wenden des Blickes nach der Seite und zugleich nach unten oder oben die beiden Balken des Kreuzes sich gegeneinander zu drehen, eine Folge der verschiedenartigen Projektion des Nachbildes.

Stelle ich mich so vor ein Kreuz, dass bei Primärstellung des Auges der horizontale Balken in dem mittleren Querschnitt der Netzhaut sich abbildet, der vertikale in dem mittleren Längsschnitt, dann entsteht das objektive Substrat für das Nachbild an eben diesen Stellen der Netzhaut. Wird jetzt das Auge gedreht, so kann der horizontale Schenkel des Nachbildes nur in der Ebene des mittleren Querschnittes, der vertikale nur in der Ebene des mittleren Längsschnittes gesehen werden, und das Nachbild wird dahin lokalisiert, wo die genannten Ebenen sich mit der Wand, auf der das Nachbild erscheint, schneiden.

Wie die Schnittfigur aussieht, lässt sich durch das Modell einfach ermitteln.

Auf der an der Vorderseite des Fusses angebrachten ebenen Fläcbe $K$ lässt sich mit einer Schraube und zwei seitlichen Stiften eine Glasplatte in vertikaler Richtung anbringen. Dieselbe ist durch vertikale und horizontale Linien in Quadrate eingeteilt und zeigt ausserdem von dem Blickpunkt bei Primärstellung ausgehend zwei diagonal verlaufende Linien.

Gibt man zunächst dem Auge die Primärstellung, schiebt die Stahlstäbchen bis an das Glas und betrachtet durch die Glasplatte lindurch ihre Enden, so sieht man, dass dieselben ein rechtwinkliges Kreuz darstellen, weil sich die beiden durch die Stahlstäbchen dargestellten Ebenen mit der Ebene der Glasplatte in einer rechtwinkligen Figur schneiden. Wird jetzt das Auge gerade nach oben, unten oder seitlich gedreht, wobei auch wieder jedes Stäbchen so weit vorgeschoben werden muss, dass es die Glasplatte berührt, daun sieht man auch wieder eine rechtwinklige Schnittfigur.

Sobald jedoch eine Drehung nach oben resp. unten und zugleich nach der Seite ausgeführt wird, bilden die an die Glasplatte anstossenden Enden der Stäbchen ein schiefwinkliges Kreuz. 
Würde man bei dem beschriebenen Versuch nicht einer ebenen Mauer, sondern einer konkav sphärisch gekrümmten Wand gegenüberstehen, so dass sich der Kopf gerade im Kugelmittelpunkt befindet, dann hätte das Nachbild überall die gleiche (richtige) Gestalt. Denn die Kugelfläche stände stets senkrecht zur Blickrichtung, wie auch das Auge sich bewegte.

Um sich auf einfachste Weise von dieser Tatsache zu überzeugen, braucht man nur in grösserer Entfernung von dem Auge eine ebene Fläche, etwa einen starken Karton, so im Raume zu orientieren, dass derselbe bei der Blickrichtung nach schräg oben senkrecht zu der Blicklinie steht. Richtet man das Auge mit dem Nachbild des Kreuzes gegen diese Fläche, dann sieht man ein rechtwinkliges Kreuz.

Um denselben Versuch auch am Modell ausführen zu können, wird die ebene Glasplatte, welche an der Fläche $K$ des Fusses $A$ angeschraubt ist, durch ein Kugelsegment aus Glas ersetzt. Die Entfernungen sind so gewählt, dass der Drehpunkt des Auges genau in das Zentrum der Kugel zu liegen kommt. Wird jetzt das Auge gedreht, so bilden bei allen Stellungen, wie man sich leicht überzeugen kann, die beiden Schnittlinien des mittleren Längs- und Querschnittes mit der Kugelfäche stets eine rechtwinklige Figur.

Helmholtz ${ }^{1}$ ) untersuchte die scheinbaren Lagen der Nachbilder von horizontalen und vertikalen Linien bei verschiedenen Augenstellungen. Die dnreh diese Nachbilder gebildeten Linien stellen Hyperbeln dar.

Man kann sich - allerdings ein wenig schematisiert - die Augenbewegung, die sich dabei abspielt, mit dem Modell folgendermaassen klarmachen.

Alle Nadeln, mit Ausnahme der in der senkrechten Reihe stehenden, werden zurückgeschoben. Diese letzteren stellen den mittleren Längsschnitt, in welchem das Nachbild eines senkrechten Streifens liegt, vor. Hierauf dreht man das Auge aus der Primärstellung gerade nach einer Seite um die Achse $F F$ und führt von dieser Stellung ausgehend Drehungen um die horizontale Achse $D D$ (Fig. 2) aus. Die Blicklinie beschreibt dann um die Achse $D D$ als Rotationsachse einen Kegelmantel, der von dem mittleren Längsschnitt tangiert wird. Der auf diese Weise zustande kommende Kegelmantel wird von der Glasplatte, welche die ebene Wand darstellt, auf die

1) H. v. Helmholtz, Handbuch der physiol. Optik, 2. Aufl, S. 621. 1896. 
projiziert wird, geschnitten, und zwar in unserem Falle in Form einer Hyperbel.

Das gleiche lässt sich für den Netzhauthorizont durch Bewegungen in den Gelenken $D D$ und $B$ zeigen.

Die Art des Kegelschnittes hängt natürlich von der Lage der Wand $a b$, auf die wir das Nachbild projizieren. Um deshalb von der zufälligen Projektion unabhängig zu sein, empfiehlt Hering ${ }^{1}$ ) zur Feststellung, ob eine Rollung des Auges stattfindet, während der Blick sich entlang einer im Raume gelegenen geraden Linie fortbewegt, den zur Erzeugung des Nachbildes dienenden Streifen in diese Linie selbst zu legen.

Aus dem gleichen Grunde erzeugte sich Helmholtz ${ }^{2}$ ) das Nachbild eines schräg stehenden Kreuzes, um zu zeigen, dass das Auge aus der Primärstellung in jede andere, also auch in eine schräge ohne Rollung übergeht.

Ich denke mir durch einen Punkt, welcher bei der Primärstellung des Auges fixiert wird, eine Gerade gezogen, so dass dieselbe mit dem Fussboden einen Winkel von $45^{\circ}$ bildet, und in dem Fixationspunkt ein rechtwinkliges Kreuz, dessen einer Schenkel mit der schrägen Linie zusammenfällt, während der andere senkrecht auf ihr steht. Wird von diesem Kreuz ein Nachbild erzeugt, und bewegt sich dann das Auge, so dass der Blickpunkt längs der erwähnten Linie gleitet, dann bleibt der eine Schenkel immer in der Leitlinie liegen, der andere bleibt immer senkrecht dazu. Aus dieser Erscheinung lässt sich schliessen, dass das Auge bei der beschriebenen Bewegung keine Rollung ausführt. Blickt man aber mit dem Nachbild des schräg stehenden, rechtwinkligen Kreuzes gerade nach oben oder gerade nach der Seite, dann tritt eine Drehung der Schenkel gegeneinander ein, und zwar im ersteren Falle so, dass der obere und untere Winkel zu spitzen, die beiden seitlichen Scheitelwinkel zu stumpfen werden, im zweiten Falle umgekehrt.

Die Erklärung ist wieder die gleiche wie bei der verzerrten Projektion des geraden Kreuzes. Die schrägen Schenkel bilden sich beim Fixieren in der Primärstellung ab auf den Schnittlinien zweier Ebenen mit der Netzhaut, welche mit der horizontalen und vertikalen

1) E. Hering, Der Raumsinn und die Bewegungen des Auges. Hermann's Handb. d. Physiol. Bd. 3 S. 343 (487). 1879.

2) H. v. Helmholtz, Handb. d. physiol. Optik, 2. Aufl., S. 622. 1896. 
Trennungslinie Winkel von $45^{\circ}$ bilden. Das Náchbild muss deshalb auch wieder in diesen Ebenen liegen, und seine Gestalt hängt $a b$ von den Schnittlinien dieser Ebenen mit der Fläche, auf der das Nachbild erscheint.

Auch diese Frage ist durch das Modell wieder leicht zu beantworten. Dazu werden die den mittleren Längs- und Querschnitt darstellenden Stäbe zurückgezogen mit Ausnahme des mittleren, welcher der Blicklinie entspricht, dafür aber die vier anderen Stäbe vorgeschoben, welche mit der Blicklinie zusammen die zwei Ebenen darstellen, in denen die Schenkel des schief stehenden Kreuzes liegen.

Lässt man nun die Blicklinie längs einer Diagonalen über die Glasplatte gleiten, dann beschreiben die bis an die Platte vorgeschobenen Enden der vier erwähnten Stäbe stets eine rechtwinklige Figur. Dreht man aber die das Auge darstellende Scheibe $G$ aus der Primärstellung gerade nach oben oder gerade nach der Seite, dann treten die beschriebenen Veränderungen des Kreuzes ein.

Anders verhält sich jedoch die Sache, wenn das Auge aus einer Sekundärstellung in eine andere Sekundärstellung übergeht. Wie theoretisch aus dem Listing'schen Gesetz folgt, und wie sich exexperimentell zeigen lässt, führt dabei das Auge Rollungen um die Blicklinie aus. Um sich davon zu überzeugen, in welcher Weise die Rollungen zustande kommen, erzeugte sich Hering ${ }^{1}$ ) in Primärstellung das Nachbild eines kurzen vertikalen Streifens, richtete den Blick nach einem seitlich gelegenen Punkte und wendete von da aus entlang einer senkrechten geraden Linie den Blick nach oben und unten. Dabei bleibt das Nachbild nicht auf der geraden Linie liegen, sondern bildet je nach der Lage einen bald grösseren, bald kleineren Winkel mit ihr.

Man kann auch diese Erscheinung mit dem Modell zeigen. Dazu braucht nur das Auge um die vertikale Achse $F F$ so nach der Seite gedreht zu werden, dass der durch die Stahlstäbe markierte mittlere Längsschnitt sich in einer der vorgezeichneten vertikalen Linien mit der Glasscheibe schneidet. Wird hierauf das Auge in der Achse $D D$ so gehoben, dass die durch die mittlere Nadel angedeutete Blicklinie auf der vertikalen Linie der Glasplatte bleibt, dann bildet die durch die Stäbe angedeutete Ebene mit der Linie der Glasplatte Winkel.

1) E. Hering, Der Raumsinn und die Bewegungen des Auges. Hermann's Handb. d. Physiol. Bd. 3 S. 343 (487). 1879. 
330 Adolf Basler: Ein Modell, welches die bei bestimmten Stellungen etc.

Das eben beschriebene Modell hat hauptsächlich den Zweck, allgemeinverständlich den Einfluss der Projektion von Nachbildern auf eine ebene Wand zu demonstrieren, und unterscheidet sich dadurch von dem Phänophthalmotrop von Donders ${ }^{1}$ ) und dem Blemmatotrop von Hermann ${ }^{2}$ ).

1) F. C. Donders, Die Bewegungen des Auges, verauschaulicht durch das Phaenophthalmotrop. Gräfe's Archiv für Ophthalmologie Bd. 16 Abt. I S. 154.1870 .

2) L. Hermann, Ein Apparat zur Demonstration der aus dem Listing schen Gesetz folgenden scheinbaren Raddrehungen. Pflüger's Arch. Bd. 8 S. 305. 1874. 